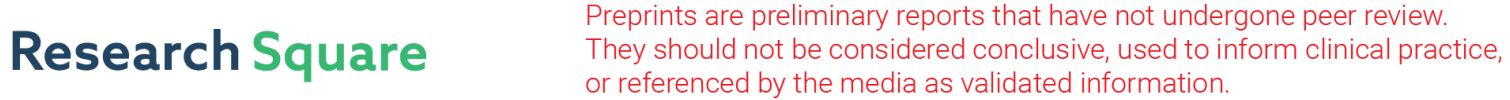 \\ An Ultrathin Graphite Based Absorber for Wearable Applications
}

Avinash ( $\nabla$ er.avinash_singh@yahoo.com )

BIT Mesra: Birla Institute of Technology https://orcid.org/0000-0003-1290-0869

Nisha Gupta

BIT Mesra: Birla Institute of Technology

\section{Research Article}

Keywords: Frequency selective surface, Microwave absorber, Ultrathin, Graphite, Flexible substrate

Posted Date: June 3rd, 2021

DOl: https://doi.org/10.21203/rs.3.rs-480007/v1

License: (c) (1) This work is licensed under a Creative Commons Attribution 4.0 International License. Read Full License 


\title{
An Ultrathin Graphite Based Absorber for Wearable Applications
}

\author{
Avinash ${ }^{1}$, Nisha Gupta ${ }^{2}$ \\ ${ }^{1}$ Department of Electronics and Communication Engineering, Birla Institute of \\ Technology, Mesra, Ranchi-835215, India \\ ${ }^{2}$ Department of Electronics and Communication Engineering, Birla Institute of \\ Technology, Mesra, Ranchi-835215, India
}

\begin{abstract}
In this paper, a low-cost, flexible, ultrathin absorber comprising a simple square patchshaped frequency selective surface (FSS) is proposed for wearable applications. The FSS based absorbing structure contains periodic graphite patch pattern placed on a thin paper substrate, and backed with a copper foil-based ground plane. The dimension and periodicity of the unit cell are $0.31 \lambda_{0}$ and $0.36 \lambda_{0}$ respectively, while the thickness of the absorber is approximately $\lambda_{0} / 85$, where $\lambda_{0}$ is the wavelength of free space at $10 \mathrm{GHz}$. The characteristics of the FSS absorber is optimized in terms of unit cell periodicity, and dielectric substrate height using a full-wave high frequency structure simulator software (HFSS). The effects of the parameters are also examined using equivalent circuit approach. The proposed absorber shows insensitivity towards the angle of incidence as well as angle of polarization. Finally, a prototype model is fabricated, measured, and validated with simulation results. A good agreement is depicted between simulated and experimental models. The proposed absorber finds application in the field of wearable electronics, medical, and automotive applications for effective mitigation of the EMI.
\end{abstract}

\section{Keywords}

Frequency selective surface, Microwave absorber, Ultrathin, Graphite, Flexible substrate. 


\section{Introduction}

In the present wireless communication scenario, due to tremendous increase in the wireless devices, the environment is full of electromagnetic (EM) pollution generated by different source of EM wave. Hence, the issues of electromagnetic interference (EMI), and electromagnetic compatibility (EMC) need more attention as both generating interference to others and also working satisfactorily in the EM environment are equally important. Among the two techniques of handling interference such as use of metallic barrier or use of EM absorbers, the later one employing light-weight flexible structure is highly preferred due to its sole purpose to absorb EMI unlike the metallic rigid structure which only diverts the EMI to other direction. An EM absorber is mostly a periodic array of metallic patterns printed over a dielectric sheet backed with a ground plane. In contrast to the traditional rigid dielectric sheets, selecting a flexible dielectric material such as paper makes the absorber thin, flexible, and conformal, thus suitable for wearable applications.

\section{Why Graphite?}

The loss parameter of the substrate as well as that of the printed conducting material plays an important role in realizing an effective thin absorber. Graphite being a low cost, lightweight, electrical conducting material of low conductivity, high complex permittivity may be preferred because it exhibits large dielectric loss owing to the high imaginary part of its permittivity [1]. The traditional conducting material on the other hand are highly conducting materials with low dielectric loss. Flexible EM absorbers can easily be realized using graphite FSS periodic pattern of PGS from Panasonic is placed over a thin dielectric sheet such as flexible paper. The PGS is a very lightweight and thin polymer sheet that exhibits excellent mechanical strength, high flexibility, and very high thermal conductivity, which makes it suitable for wearable outdoor applications. Flexible sheet can be easily cut into desired shapes. Due to its high thermal conductivity, it is mostly used for managing the heat in electronics circuitry. Moreover, the sheet resistance can easily be modified to the desired value by changing the applied voltage bias, thus dictating its future application in several tuneable device applications. 


\section{Related Works}

Design of low cost, thin and conformal EM absorber is indeed a complicated task [2]. Selection of materials of both substrate as well as FSS pattern are equally important in deciding the absorption characteristics of the EM absorber. Design of a thin lightweight conformal absorber is still a challenge, and a large number of researchers have attempted to overcome this challenge while designing the EM absorbers. The design of a resonant Salisbury absorber with optimum resistive surface is presented in [3]. However, the problem lies with the thickness of spacer between ground plane and resistive surface. Among the various techniques employed to overcome thickness problem in designing the EM absorber, use of lossy FSS technique [4], high impedance surface technique [5], circuit analog absorber [6] are noteworthy. While the basic principle of realizing a thin EM absorber is discussed in [7], a thin capacitive surface based absorber is explained in [8]. A very thin, flexible pyrolytic graphite sheet (PGS) made from highly oriented graphite polymer film is selected for the FSS in [9]. In the past graphite is also used in the design of patch antennas where the conventional conducting patch is replaced by graphite patch [1]. However, the high resistivity of the material leads to poor performance of the antenna in terms of gain and radiation efficiency. On the other hand, high resistivity of the graphite offers advantage in the design of absorber, where the high sheet resistance enhances the absorption mechanism of the absorber. Use of graphite sheet alone for attenuating the microwave radiation is also reported by some of the physicists [10-11]. In the present work, instead of using graphite sheet alone for mitigating the EMI, it is used as a conducting material for the design of the FSS patch. The design of a simple square graphite patch FSS absorber is proposed in this work and based on the characteristics of the material and the extensive simulation parameters, a scheme is proposed for realization of a thin EM absorber at $10 \mathrm{GHz}$.

\section{Design and Analysis of Absorber}

The proposed absorber is different from a traditional absorber both in terms of material and the fabrication process. Hence, instead of altogether proposing a new FSS patch design 
to demonstrate the design and fabrication process, a well-known, and thoroughly investigated square patch FSS is considered. Design and analysis of a square patch FSS for its application as EM absorber is systematically explained in [5], [12], [13]. Fig.1 shows a unit cell of the proposed square patch array of PGS (conductivity, $\sigma=2 \times$ $10^{6} \mathrm{~S} / \mathrm{m}$ ) absorber of $0.025 \mathrm{~mm}$ thick FSS layer on the top of a dielectric layer. Two layers of a paper sheet of thickness $0.177 \mathrm{~mm}\left(\varepsilon_{r}=2.2, \boldsymbol{\delta}_{\boldsymbol{t}}=0.05\right)$ is used as dielectric substrate grounded on a copper foil with thickness $0.011 \mathrm{~mm}$ (conductivity, $\sigma=5.8 \times$ $\left.10^{7} \mathrm{~S} / \mathrm{m}\right)$.

Impedance matching of the EM wave (free space impedance) impinging on the surface of the absorber with that of the absorber is the first and foremost criteria in the design of the EM absorber. Moreover, this phenomenon can be very well explained by adopting the equivalent circuit model of the absorber, where each layer of the absorber is depicted clearly and perfect matching can be achieved by selecting specific set of FSS patch parameters. The surface current distribution approach [14] is applied to draw the equivalent circuit of the proposed absorber.

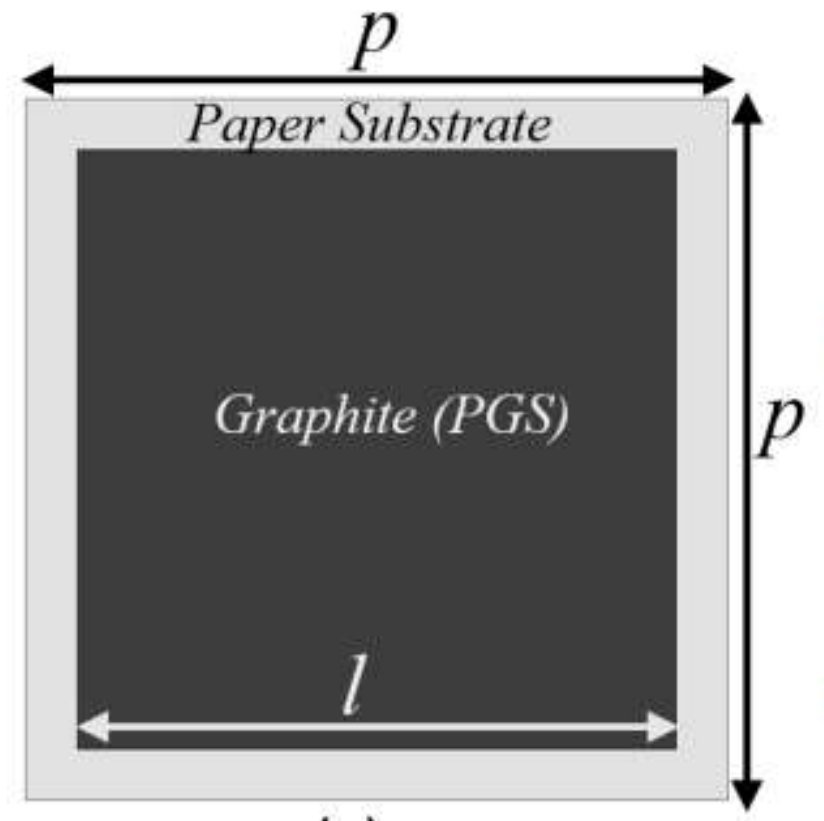

(a)

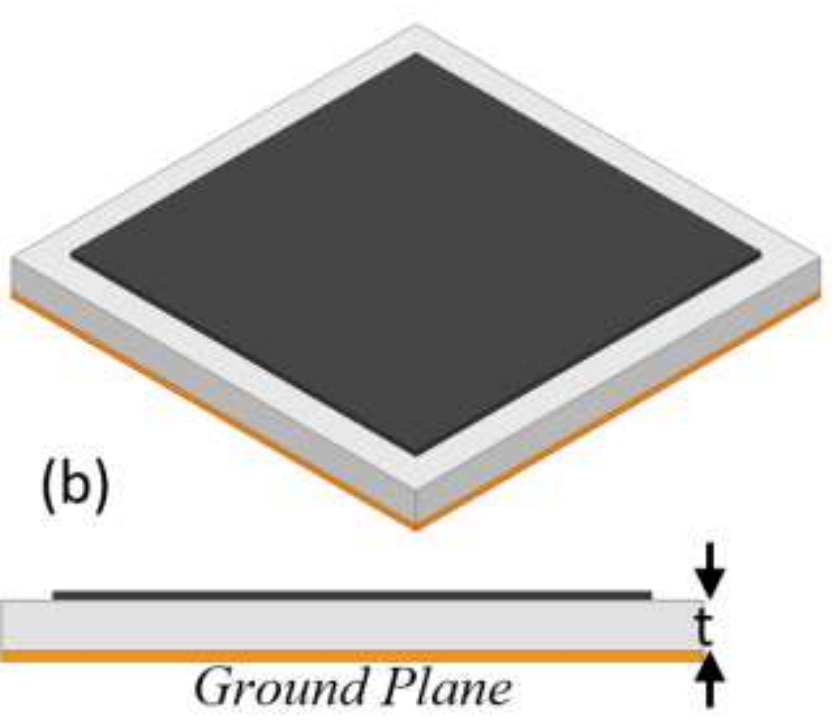

(c)

Fig.1 Unit cell of proposed absorber with dimensions (a) Top view: Periodicity of the absorber, $p=10.9 \mathrm{~mm}$, length of the square patch length, $l=9.3 \mathrm{~mm}$ (b) Isometric View and (c) Side View, thickness of substrate, $t=0.354 \mathrm{~mm}$ 
In Fig.2 The equivalent circuit model of the proposed absorber is presented where a series RLC circuit represents the unit cell of the FSS. The impedance of the FSS, $Z_{F S S}$ can be represented as

$$
Z_{F S S}=R_{F S S}+j \omega L_{F S S}+\frac{1}{j \omega C_{F S S}}
$$

Where, $R_{F S S}, L_{F S S}, C_{F S S}$ are the equivalent resistance, inductance and capacitance of the FSS and $\omega$ is angular frequency of the incident EM wave.

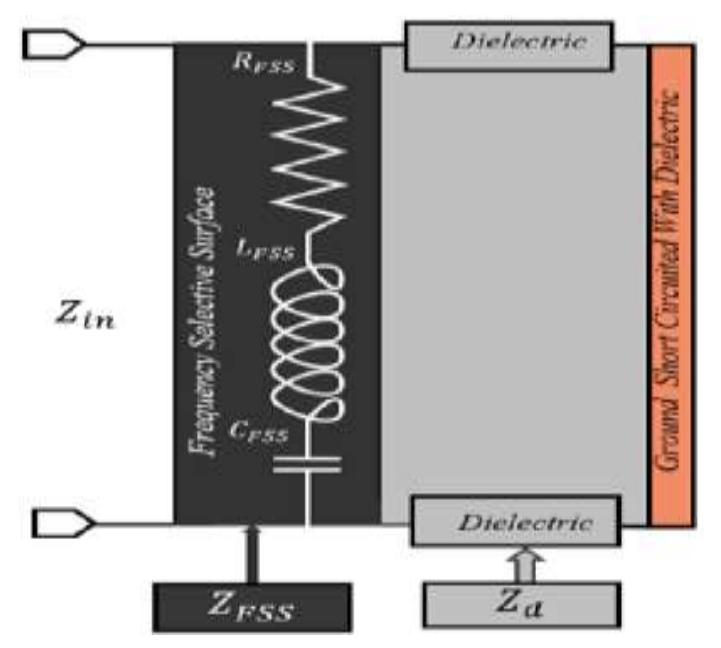

Fig.2 Equivalent circuit of the unit cell of proposed absorber

Various techniques such as analytical and simulation-based approach [15-16], can be used to extract equivalent circuit parameters of the proposed absorber. The extracted values of $R_{F s S S}, L_{F S S}$, and $C_{F S S}$ are $1.19 \Omega, 0.165 \mathrm{nH}$, and $0.377 p F$ respectively.

The impedance of a dielectric slab can be considered as piece of a transmission line with input impedance $Z_{d}$, and can be given as

$$
Z_{d}=Z_{0}\left(\frac{Z_{L}+j Z_{0} \tan (\beta d)}{Z_{0}+j Z_{L} \tan (\beta d)}\right)
$$

A highly conductive metal foil is used as a ground plane of the proposed absorber, which also acts as a load for the dielectric slab. The impedance of a metal foil is almost equivalent to zero, so in $(2), Z_{L} \approx 0$, and impedance of a transmission line with a short-circuited load is represented as

$$
Z_{d}=j Z_{0} \tan (\beta d)
$$

Where, $Z_{0}$ is the intrinsic impedance, $\beta$ is phase constant and $d$ is the thickness of dielectric slab. 


$$
Z_{0}=\sqrt{\frac{\mu}{\varepsilon}}=\sqrt{\frac{\mu_{r} \mu_{0}}{\varepsilon_{r} \varepsilon_{0}}}=\frac{377}{\sqrt{\varepsilon_{r}}} \quad \text { and }, \beta=\omega \sqrt{\mu \varepsilon}
$$

The surface impedance, $Z_{\text {in }}$ of the absorber is equivalent to the parallel connection between $Z_{F S S}$ and $Z_{d}$ as

$$
Z_{\text {in }}=Z_{F S S} \| Z_{d}
$$

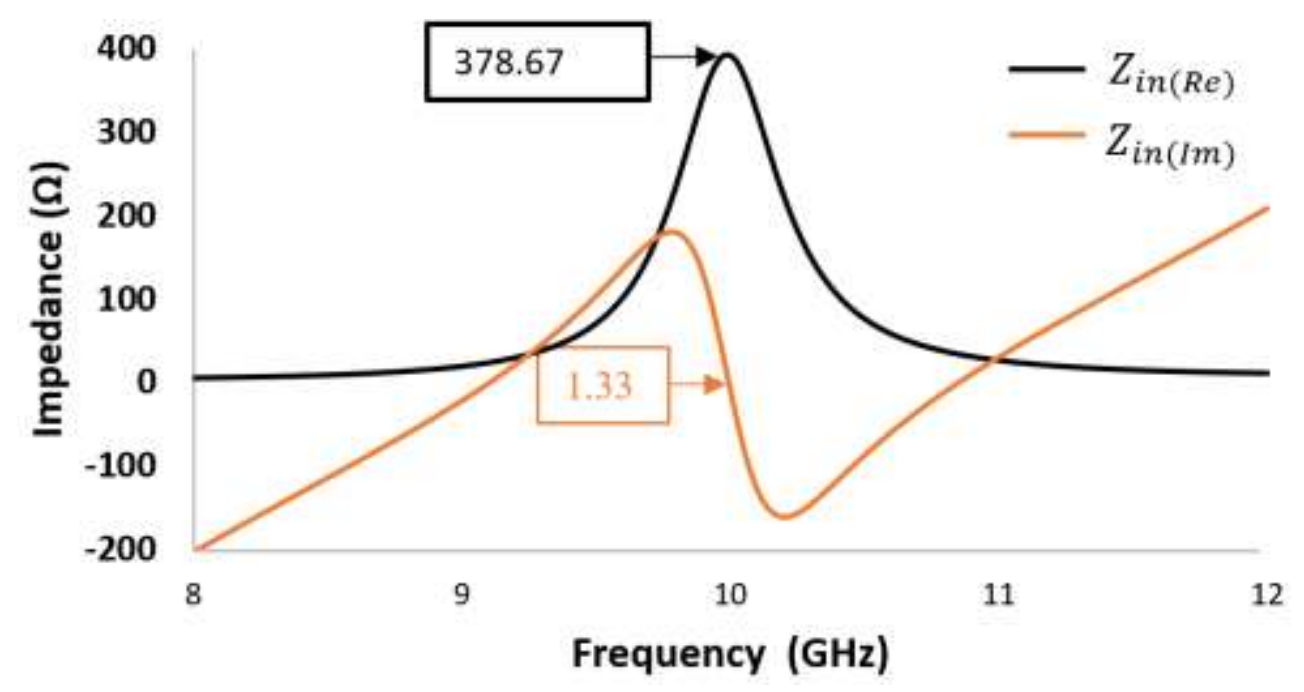

Fig. 3 Surface impedance of the proposed absorber at $10 \mathrm{GHz}$

Equivalent circuit model of the proposed absorber is simulated with the help of advanced design system (ADS) software. Fig.3 reveals that the surface impedance of the proposed absorber with $Z_{\text {in }}(\Omega)=378.67+j 1.33$ is perfectly matched $(0.41 \%$ error $)$ with respect to the free space impedance, $\eta_{0}(\Omega)=377$.

The reflection coefficient, $S_{11}$ of the absorber is calculated by

$$
S_{11}=\frac{Z_{i n}-\eta_{0}}{Z_{i n}+\eta_{0}}
$$

A highly accurate finite element method-based simulator HFSS is used to simulate the proposed absorber. The resonating frequency of $10 \mathrm{GHz}$ is used in simulation. Fig. 4. Shows the reflection coefficients obtained using ADS, HFSS as well as measurement, which confirms that the circuit parameters extracted are acceptable.

The absorptivity of the proposed absorber can be calculated as

$$
\text { Absorption }=1-\left|S_{11}\right|^{2}-\left|S_{21}\right|^{2}
$$


The ground plane being a highly conductive material copper with thickness more than its skin depth calculated at lowest frequency of $\mathrm{X}$ band, prohibits the transmission of the EM wave completely. Hence, $S_{21} \approx 0$ and absorption totally depends on the value of $S_{11}$ as

$$
\text { Absorption }=1-\left|S_{11}\right|^{2}
$$

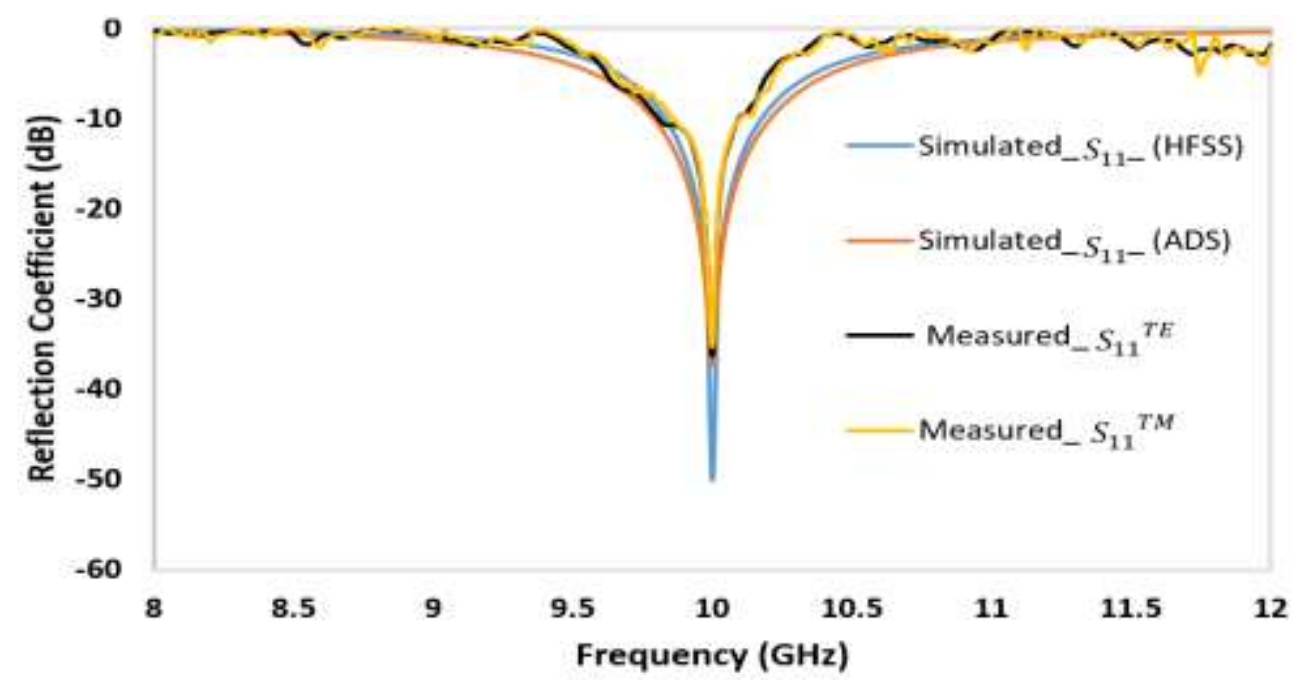

Fig.4 Reflection coefficient: comparison between simulated and measured $S_{11}$ of the proposed absorber

\section{Experimental Results and Discussion}

For verification of the proposed absorber, a prototype sample of $10 \times 10$ unit cell is fabricated on a $110 \mathrm{~mm} \times 110 \mathrm{~mm}$ planar paper with a thickness of $0.177 \mathrm{~mm}$ and two sheets are pasted back to back to make a total thickness of $0.354 \mathrm{~mm}$. A copper foil of thickness $0.011 \mathrm{~mm}$ is pasted on the back of the paper to act as a ground. The most important part of the absorber, the FSS is fabricated with the available PGS EYGS121803 by Panasonic with dimensions $115 \mathrm{~mm}$ x $180 \mathrm{~mm}$. A total of 100 graphite patches with optimized dimension of $9.3 \mathrm{~mm}$ are cut manually with a detail knife. After cutting PGS, the patches are pasted on the top of the substrate with adhesive tape manually. However, during the PGS cutting and pasting process, accuracy and precision are given prime importance, yet some errors are inevitable. A very thin lamination of the complete absorber makes it work under all weather conditions, which hardly changes the 
characteristics of the absorber. Fig.5 shows the fabricated sample of the proposed absorber.

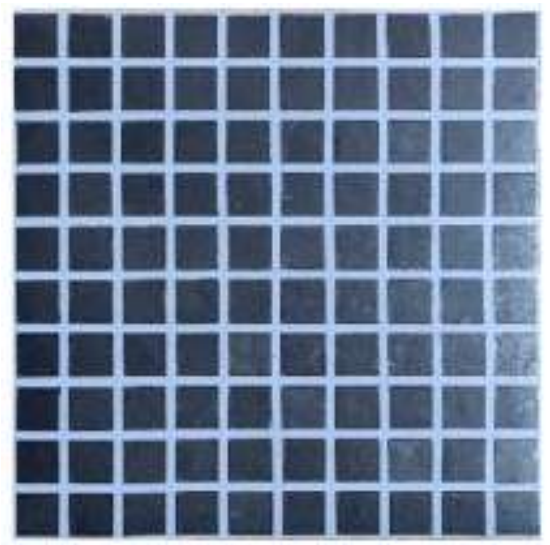

(a)

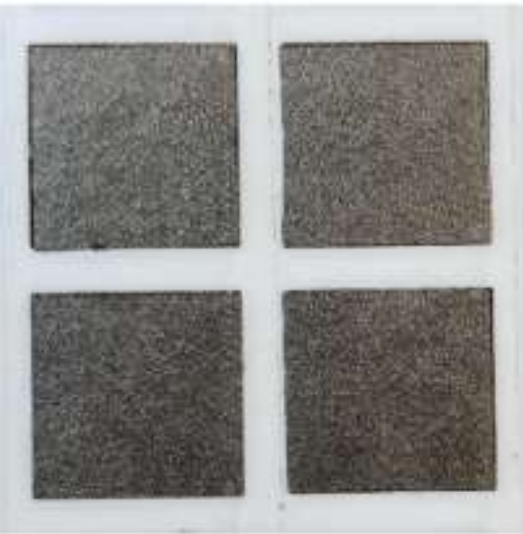

(b)

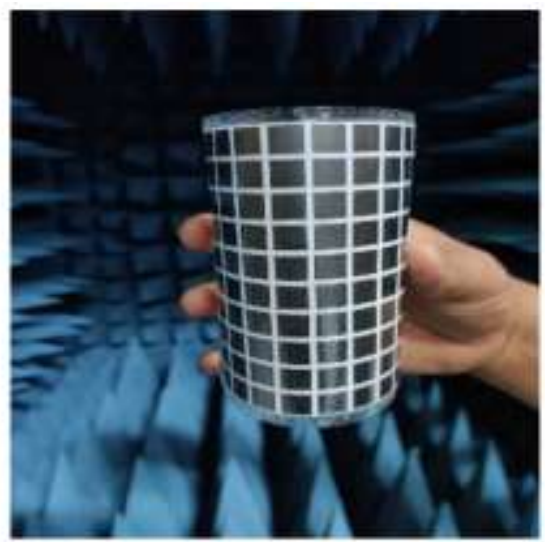

(c)

Fig.5 Fabricated prototype of proposed absorber (a) Front view (b) Expanded View and (c) Flexible view

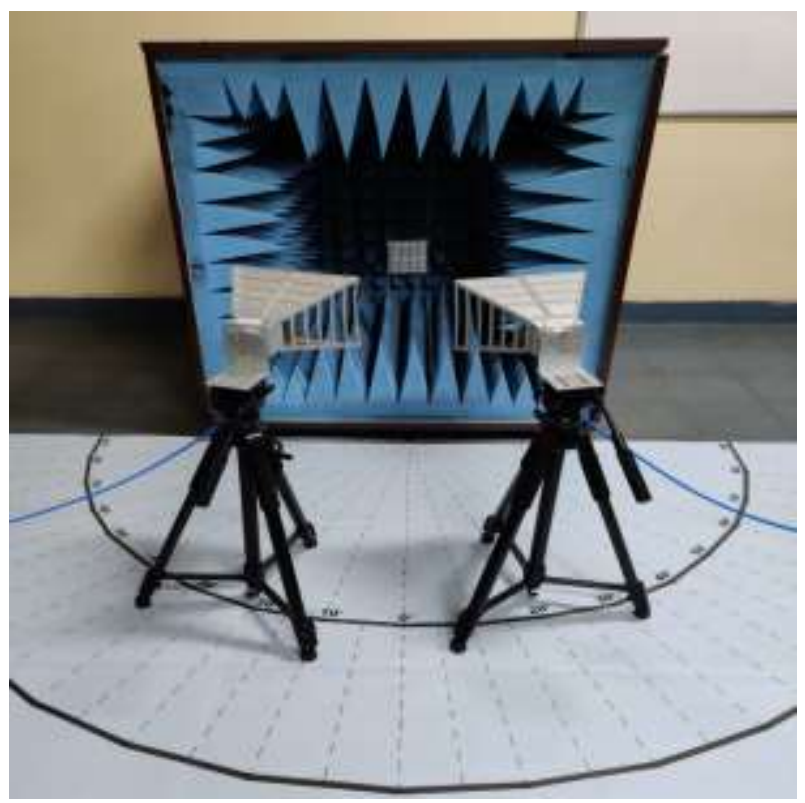

Fig.6 Experimental setup for the proposed absorber

For measurement, purposes the sample is kept inside an anechoic chamber. Two X-Band (8GHz-12 GHz) horn antennas are connected to the two ports of an Agilent Technologies N5230A (10 MHz - $20 \mathrm{GHz})$ PNA-L network analyzer. The complete setup is shown 
in Fig.6 A distance of $1.5 \mathrm{~m}$ is maintained between sample and horn antenna to measure the reflection coefficient for different angle polarization $(\varphi)$, and incident angle $(\theta)$ varying from $\varphi=0^{\circ}$ to $90^{\circ}$ and $\theta=0^{\circ}$ to $75^{\circ}$ respectively. Moving average technique is applied to smooth the measured result, was compared with the simulated one. Fig.4 indicates a reasonable agreement between the simulated and measured results of the reflection coefficient for normal incidence of TE and TM mode.

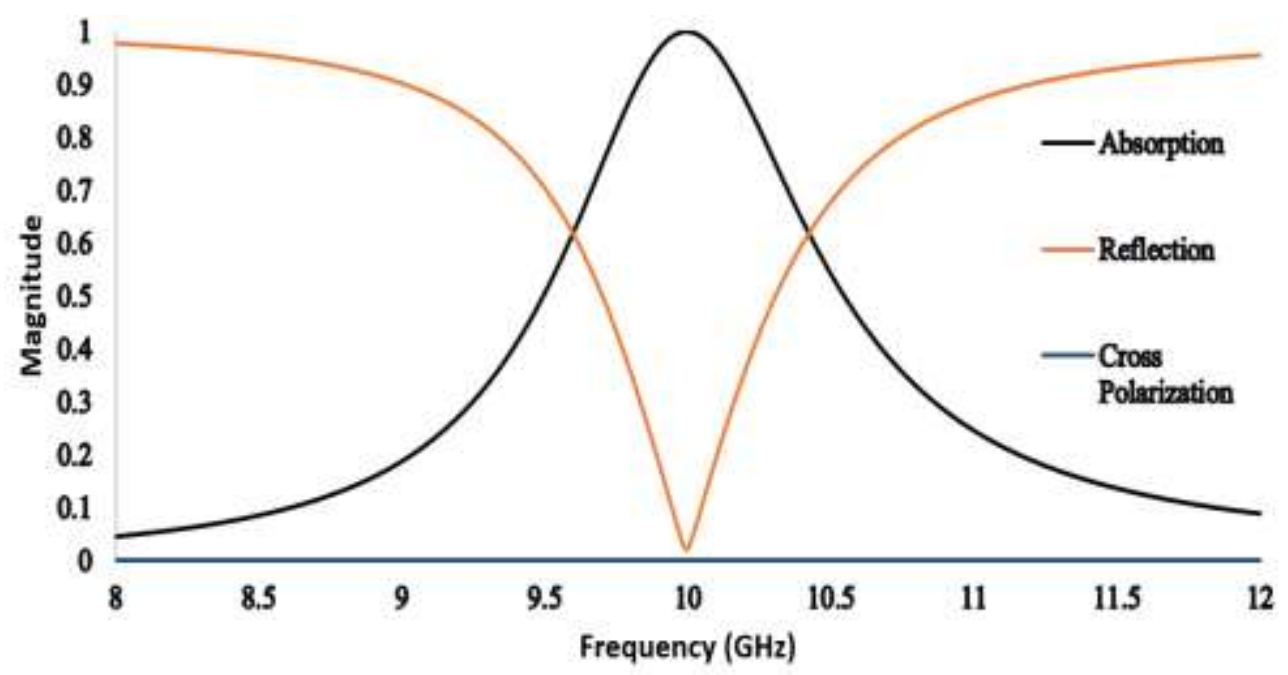

Fig.7 Simulated absorption, reflection and cross polarization of the proposed absorber

Fig.7 Shows simulated and measured magnitude of reflection, cross polarization and absorption of the proposed absorber. Almost zero reflection, zero cross polarization, and 99.99\% absorption are achieved in the proposed absorber.

To realize an absorber that behaves uniformly at any angle of polarization of EM wave, the interaction between the electric field and the structure must be steady at all angles of polarisation. Since the structure used in the FSS possess axial symmetry, the proposed absorber is angularly stable. Fig.8(a) shows that absorption remains almost constant at different polarisation angles $(\varphi)$ which makes the proposed absorber polarization insensitive. The proposed absorber is also studied for oblique incidence of TE- Mode. Fig.8(b) shows that more than $80 \%$ absorption is achieved upto $60^{\circ}$ angle of incidence $(\theta)$. 


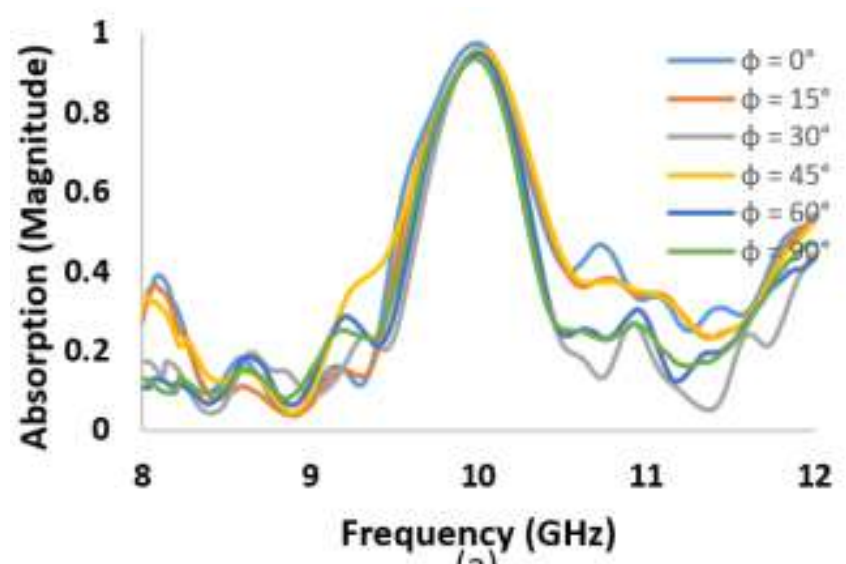

(a)

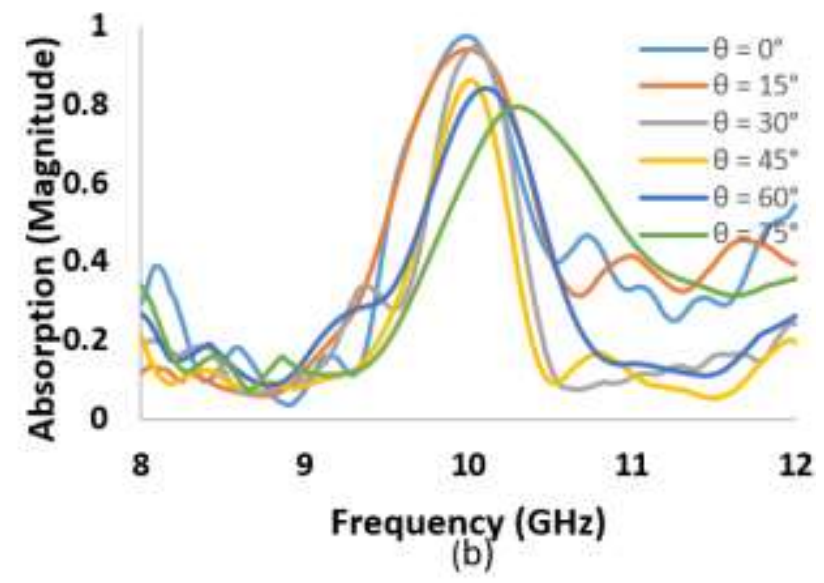

(b)

Fig.8 Simulated absorption (a) for different angle of polarization $\varphi$ and (b) for different incident angle $\theta$

At the moment when EM wave strikes on the surface of the absorber, instantly free charge carriers distribute themselves and induce an electric field. Fig.9(a) shows surface current distribution on the top surface of the proposed absorber at the resonant frequency which exhibits that most of the current is distributed on the surface of conducting patch. This can be easily explained based on the concept of skin effect. The confinement of the more current towards the edges depends on the dielectric property of the substrate, frequency of operation, and the discontinuity of the patch. Negligible displacement current also flows through dielectric layer. Fig.9(b) shows electric field distribution of the proposed absorber.

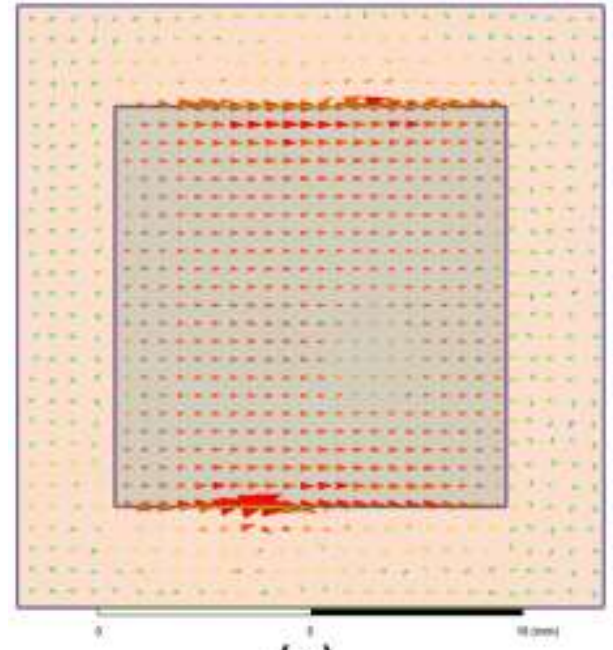

(a)

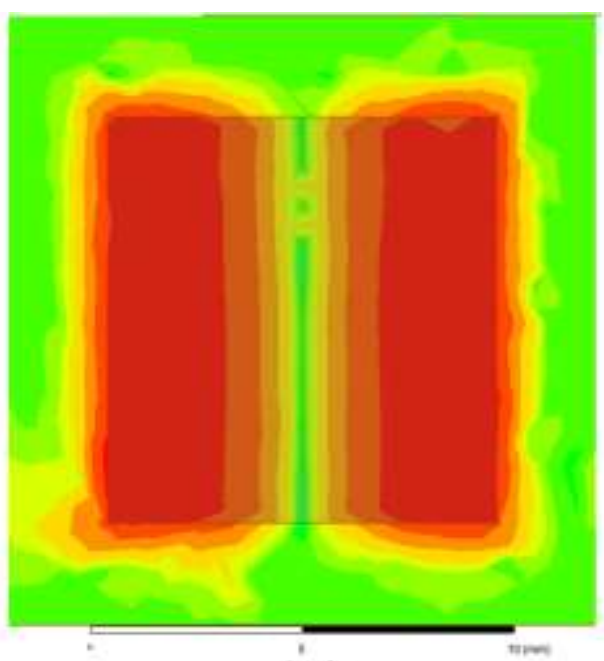

(b)

Fig.9 Simulated plot of (a) surface current distribution (b) electric field distribution of the proposed absorber at $10 \mathrm{GHz}$ 
Table 1: Comparison of various reported absorbers with proposed ultrathin absorber

\begin{tabular}{cccccccc}
\hline Ref. & $\boldsymbol{f}_{\boldsymbol{r}}{ }^{\mathbf{a}}(\mathbf{G H z})$ & Substrate & $\boldsymbol{t}^{\mathbf{b}}(\mathbf{m m})$ & $\boldsymbol{\varepsilon}_{\boldsymbol{r}}{ }^{\mathbf{c}}$ & $\boldsymbol{\delta}_{\boldsymbol{t}}{ }^{\mathbf{d}}$ & FSS Material & Flexiblity \\
\hline$[17]$ & 10.14 & Fr4 & 1 & 4.3 & 0.02 & Copper & No \\
\hline$[18]$ & 10 & Rogers4003 & 0.508 & 3.38 & 0.0027 & Copper & No \\
\hline$[19]$ & 10.8 & Textile & 1.2 & 1.8 & 0.12 & Silver Conductive Ink & Yes \\
\hline$[20]$ & 9.5 & Paper & 1.19 & 3 & 0.05 & Silver Conductive Ink & Yes \\
\hline$[21]$ & 11 & PDMS & 0.5 & 2.89 & 0.02 & Silver Conductive Ink & Yes \\
\hline Proposed & 10 & Paper & 0.354 & 2.2 & 0.05 & PGS & Yes \\
\hline \multicolumn{7}{c}{$\begin{array}{c}{ }^{a} \text { Resonating frequency } \\
\text { b Thickness of substrate } \\
\text { celative permittivity of substrate } \\
\text { d Loss tangent of substrate }\end{array}$} \\
c
\end{tabular}

Finally, the proposed FSS absorber is compared with the several other absorbers available in the literature and their performance parameters are compared in the Table 1. As seen the proposed absorber outperforms the other absorbers in terms of the thickness. Besides, a simple fabrication technique is used to develop the prototype model. For the purpose of mass production, a well-established screen-printing technology may be employed to realize the proposed absorber, where graphite ink may be used in screen printing.

\section{Conclusion}

An ultra-thin, lightweight, flexible resonant absorber is realized using low cost and readily available Panasonic graphite sheet, paper sheet, and copper foil. Electromagnetic parameters of the proposed absorber are first converted to circuit parameter to derive the equivalent circuit of the proposed absorber. The impedance matching of the proposed absorber with free space impedance, shows almost zero reflection, and $99.99 \%$ absorption. Also, the proposed absorber works perfectly for all angle of polarization and is hardly influenced by the different angle of incidence. A comparison of the proposed absorber with the previously reported absorber for X band application shows superiority of the absorber in terms of thickness, which is achieved with the help of pyrolytic graphite sheet. The proposed absorber is found to be suitable for wearable outdoor applications.

\section{Declarations}


Funding: This study was not funded by any one. Funding information is not applicable and No funding was received.

Conflict of interests / Competing interests: The authors have no conflicts of interest to declare that are relevant to the content of this articl

Availability of data and material: Work contained in this manuscript is original and whenever I have used materials (data, theoretical analysis and text) from other sources, I have given due credit to them by citing them in the text and giving their details in the references.

Code availability: The work does not require any coding as the simulation software package is used in the analysis.

Authors' contributions: The work contained in this manuscript is original. In this manuscript a low-cost graphite based thin resonant electromagnetic absorber is proposed for the wearable application. A comparison of the proposed absorber with the other existing absorbers clearly shows its benefit in terms of thickness and cost.

\section{References}

1. Ansari, A., \& Akhtar, M. J. (2017). Co/graphite based light weight microwave absorber for electromagnetic shielding and stealth applications. Materials Research Express, 4(1), 016304

2. Tirkey, M. M., \& Gupta, N. (2019). Electromagnetic absorber design challenges. IEEE Electromagnetic Compatibility Magazine, 8(1), 59-65.

3. Chambers, B. (1994). Optimum design of a Salisbury screen radar absorber. Electronics Letters, 30(16), 1353-1354. 
4. Silva, M. W. B., Campos, A. L. P. S., \& Kretly, L. C. (2015). Design of thin microwave absorbers using lossy frequency selective surfaces. Microwave and Optical Technology Letters, 57(4), 928-933.

5. Costa, F., Monorchio, A., \& Manara, G. (2010). Analysis and design of ultra thin electromagnetic absorbers comprising resistively loaded high impedance surfaces. IEEE Transactions on Antennas and Propagation, 58(5), 1551-1558.

6. Munk, B. A., Munk, P., \& Pryor, J. (2007). On designing Jaumann and circuit analog absorbers (CA absorbers) for oblique angle of incidence. IEEE Transactions on Antennas and Propagation, 55(1), 186-193.

7. Tretyakov, S. (2016). Thin absorbers: operational principles and various realizations. IEEE Electromagnetic Compatibility Magazine, 5(2), 61-66.

8. Han, Y., Che, W., Christopoulos, C., \& Chang, Y. (2015). Investigation of thin and broadband capacitive surface-based absorber by the impedance analysis method. IEEE Transactions on Electromagnetic Compatibility, 57(1), 22-26.

9. https://www.farnell.com/datasheets/2244033.pdf

10. Wu, K. H., Cheng, K. F., Wang, J. C., \& Chang, Y. C. (2017). Preparation of magnetic expanded graphite with microwave absorption and infrared stealth characteristics. Materials Express, 7(6), 500-508.

11. Borah, D., \& Bhattacharyya, N. S. (2017). Design and development of expanded graphite-based non-metallic and flexible metamaterial absorber for X-band applications. Journal of Electronic Materials, 46(1), 226-232.

12. Luukkonen, O., Simovski, C., Granet, G., Goussetis, G., Lioubtchenko, D., Raisanen, A. V., \& Tretyakov, S. A. (2008). Simple and accurate analytical model of planar grids and high-impedance surfaces comprising metal strips or patches. IEEE Transactions on Antennas and Propagation, 56(6), 1624-1632.

13. Firouzfar, A., Afsahi, M., \& Orouji, A. A. (2019). Novel synthesis formulas to design square patch frequency selective surface absorber based on equivalent circuit model. International Journal of $R F$ and Microwave Computer-Aided Engineering, 29(6), e21680. 
14. Ghosh, S., \& Srivastava, K. V. (2014). An equivalent circuit model of FSS-based metamaterial absorber using coupled line theory. IEEE Antennas and Wireless Propagation Letters, 14, 511-514.

15. Costa, F., Monorchio, A., \& Manara, G. (2009, September). An equivalent-circuit modeling of high impedance surfaces employing arbitrarily shaped FSS. In 2009 International Conference on Electromagnetics in Advanced Applications (pp. 852855). IEEE.

16. Sjöberg, D. (2008). Analysis of wave propagation in stratified structures using circuit analogues, with application to electromagnetic absorbers. European journal of physics, 29(4), 721.

17. Zhu, B., Wang, Z., Huang, C., Feng, Y., Zhao, J., \& Jiang, T. (2010). Polarization insensitive metamaterial absorber with wide incident angle. Progress In Electromagnetics Research, 101, 231-239.

18. Liu, Y., De Flaviis, F., \& Alexopoulos, N. G. (2008). A thin X-band microwave absorber using a center shorted spiral medium. IEEE Antennas and Wireless Propagation Letters, 8, 271-274.

19. Lee, D., Kim, H. K., \& Lim, S. (2017). Textile metamaterial absorber using screen printed channel logo. Microwave and Optical Technology Letters, 59(6), 14241427.

20. Yoo, M., Kim, H. K., Kim, S., Tentzeris, M., \& Lim, S. (2015). Silver nanoparticlebased inkjet-printed metamaterial absorber on flexible paper. IEEE Antennas and Wireless Propagation Letters, 14, 1718-1721.

21. Jeong, H., \& Lim, S. (2017). A stretchable electromagnetic absorber fabricated using screen printing technology. Sensors, 17(5), 1175. 


\section{Figures}

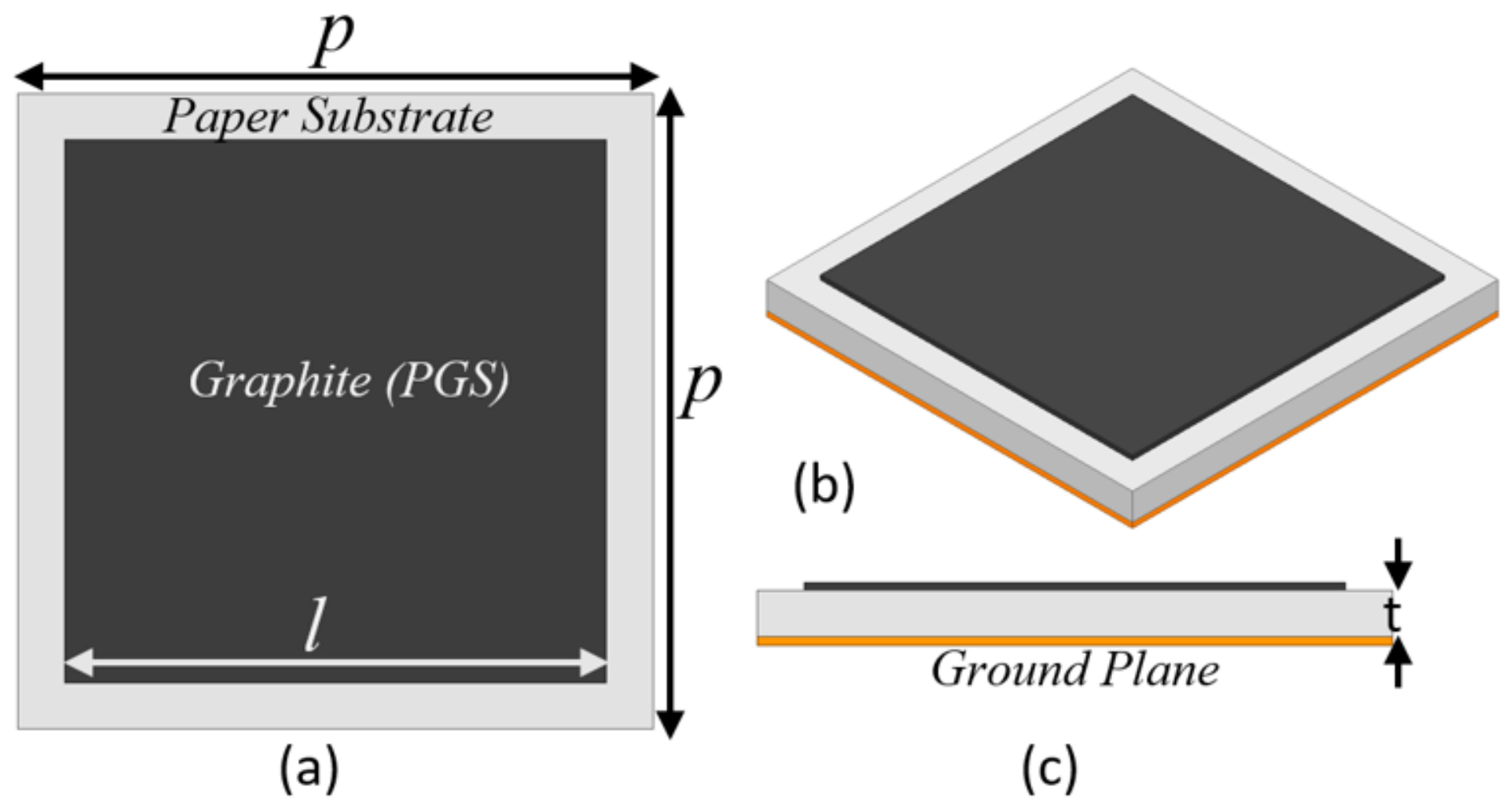

Figure 1

Unit cell of proposed absorber with dimensions (a) Top view: Periodicity of the absorber, $p=10.9 \mathrm{~mm}$, length of the square patch length, I $=9.3 \mathrm{~mm}$ (b) Isometric View and (c) Side View, thickness of substrate, $\mathrm{t}=0.354 \mathrm{~mm}$

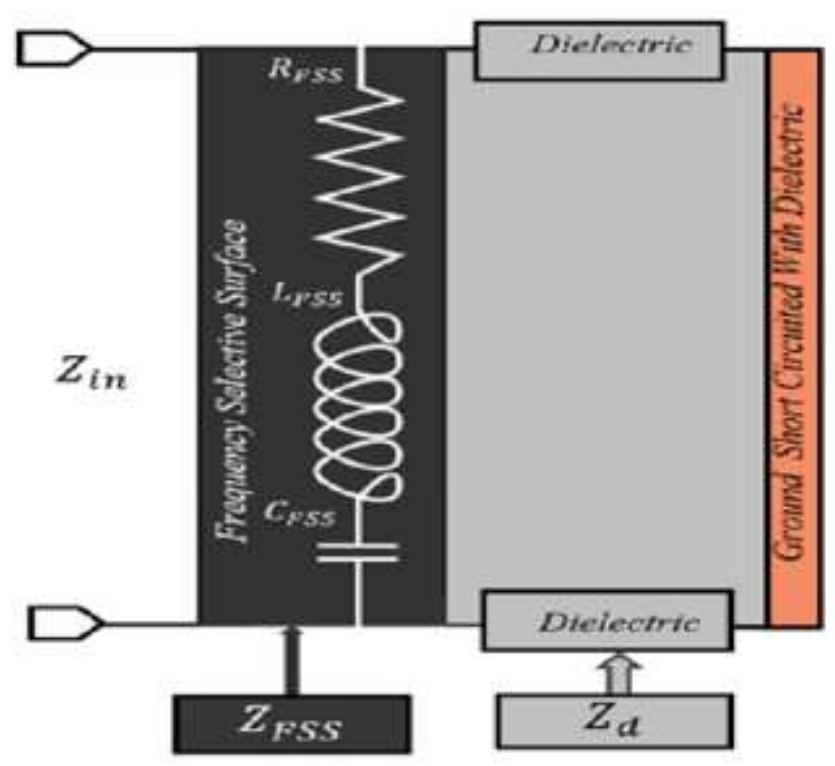

Figure 2 
Equivalent circuit of the unit cell of proposed absorber

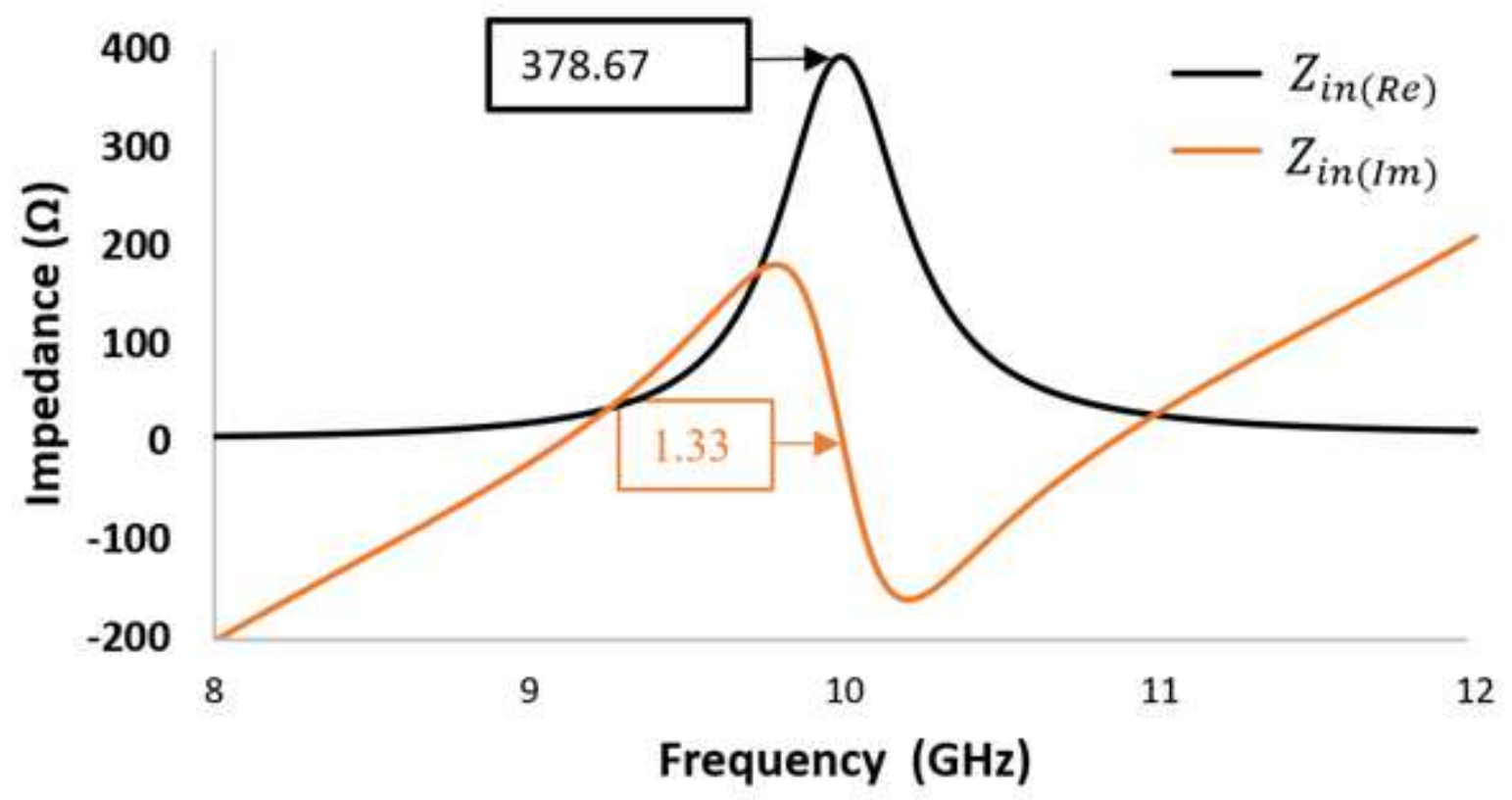

Figure 3

Surface impedance of the proposed absorber at $10 \mathrm{GHz}$

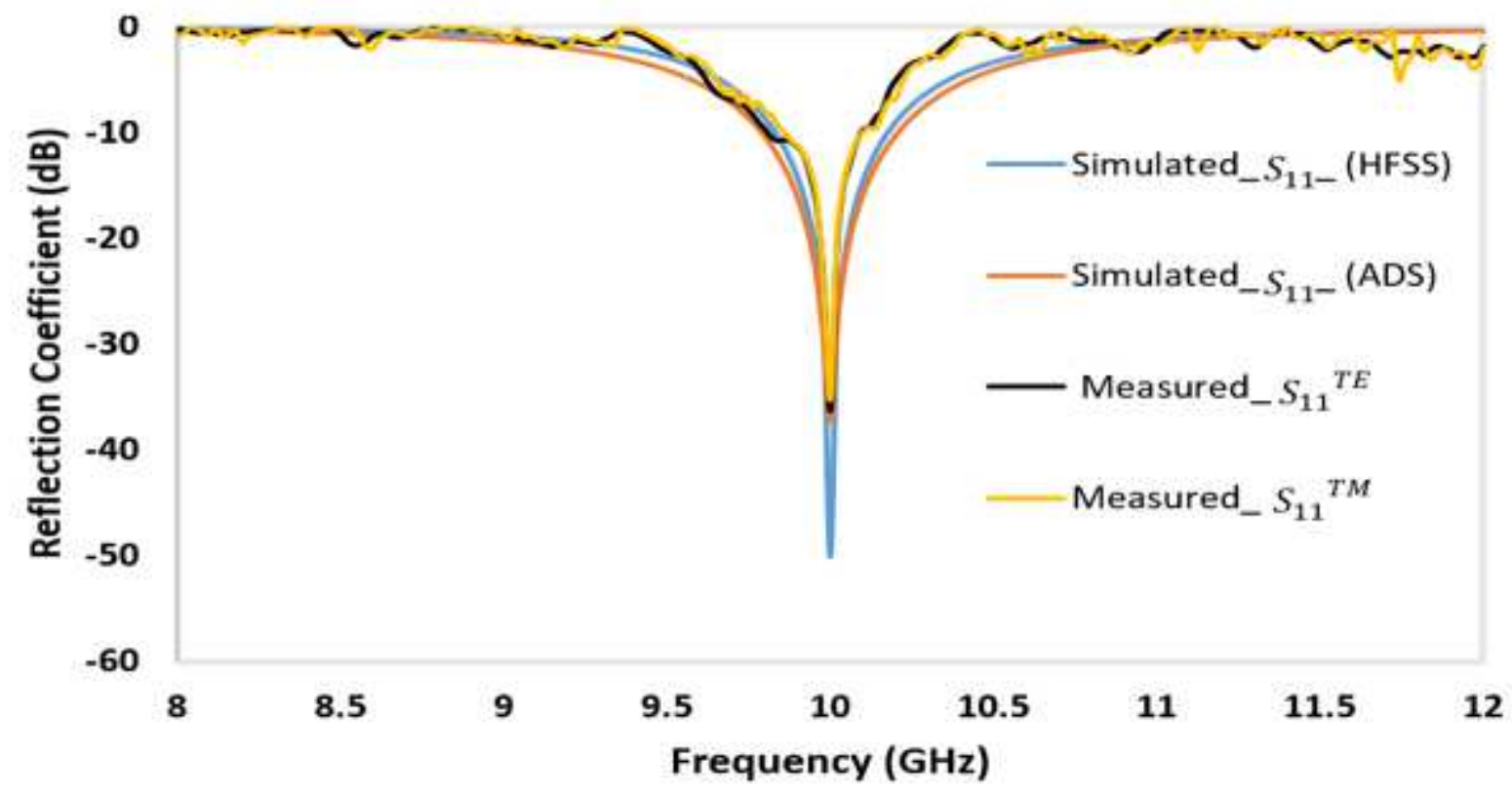

Figure 4

Reflection coefficient: comparison between simulated and measured S_11 of the proposed absorber 


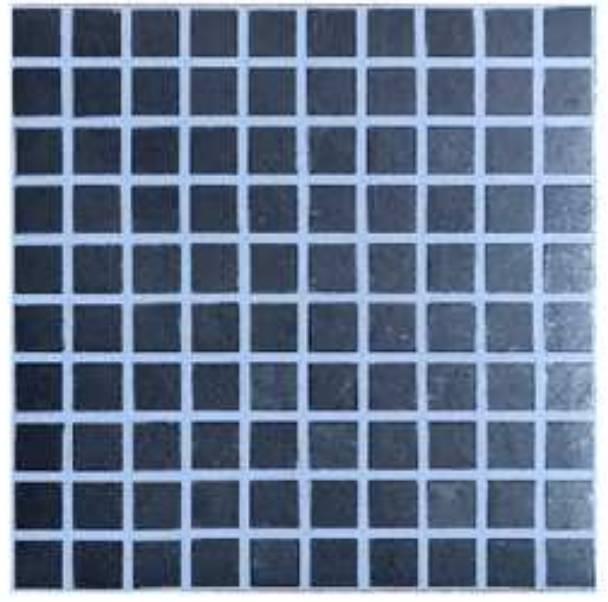

(a)
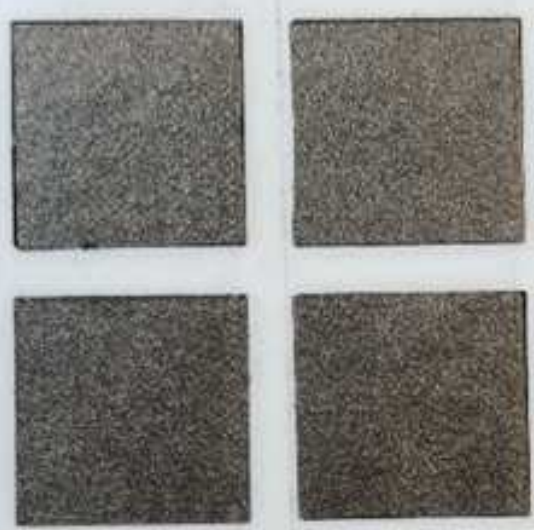

(b)

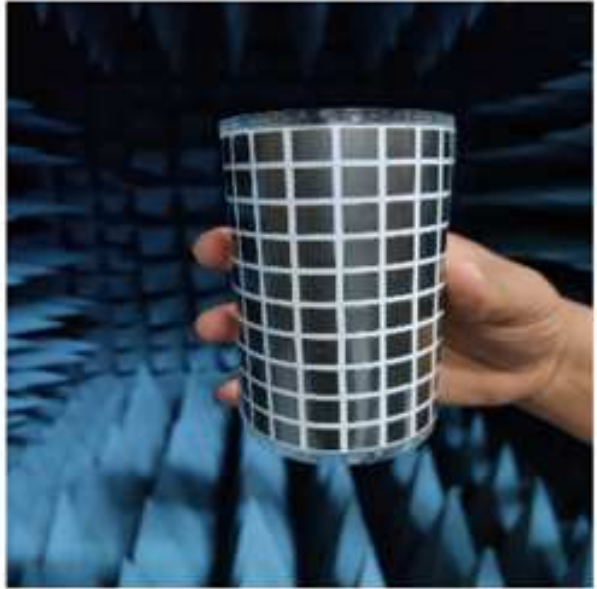

(c)

Figure 5

Fabricated prototype of proposed absorber (a) Front view (b) Expanded View and (c) Flexible view

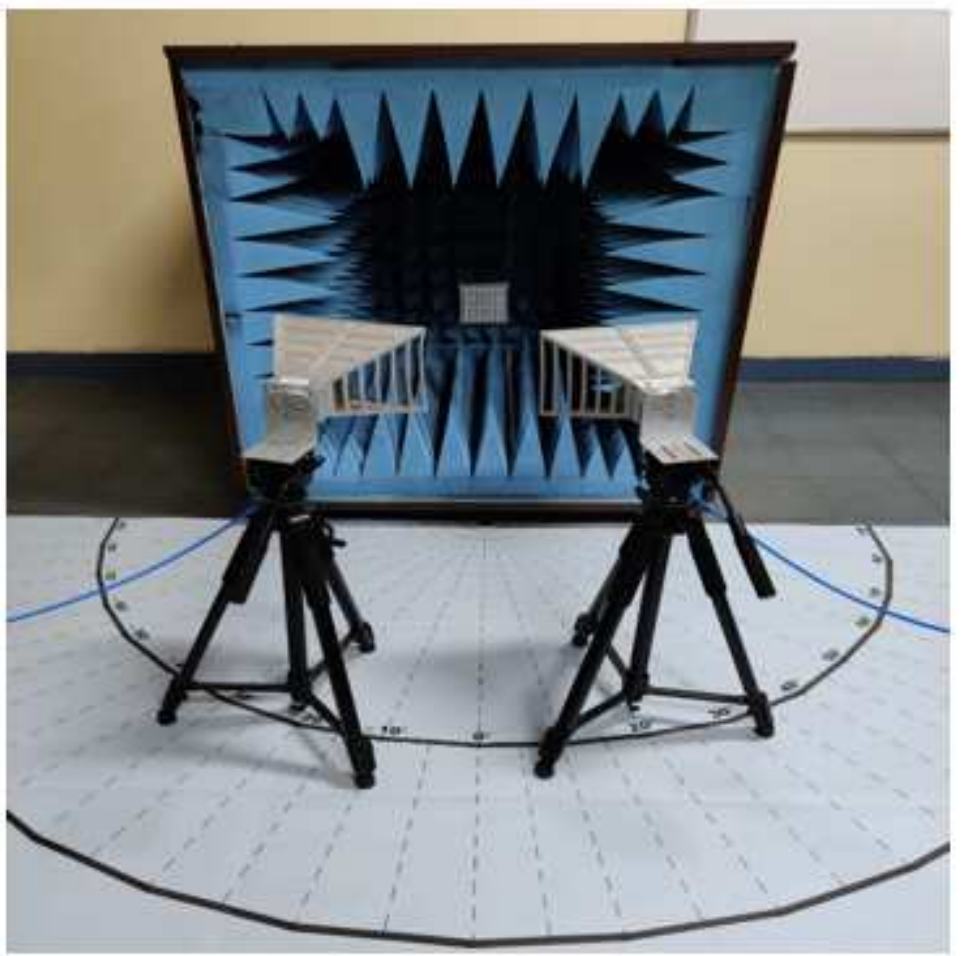

Figure 6

Experimental setup for the proposed absorber 


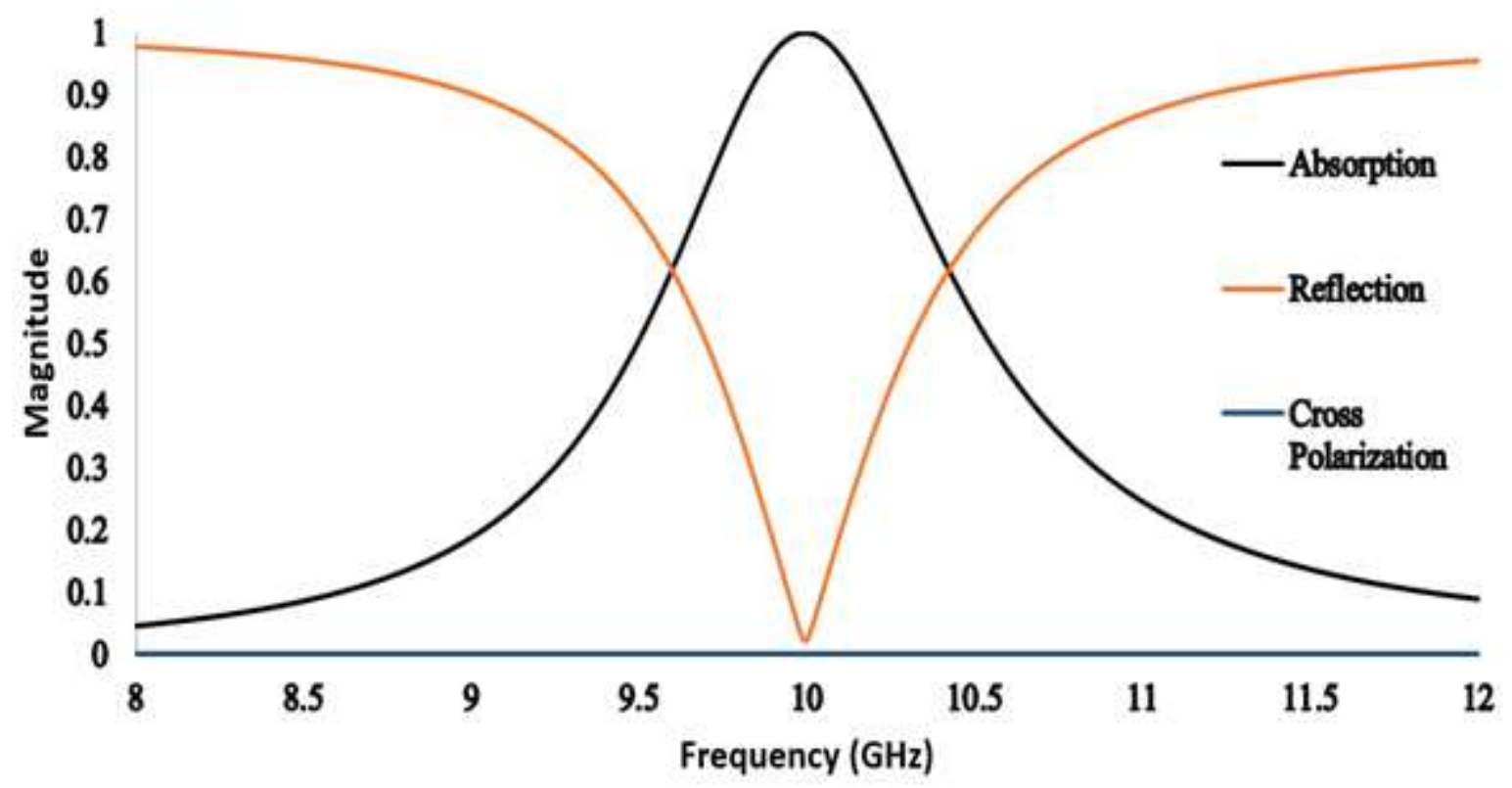

Figure 7

Simulated absorption, reflection and cross polarization of the proposed absorber
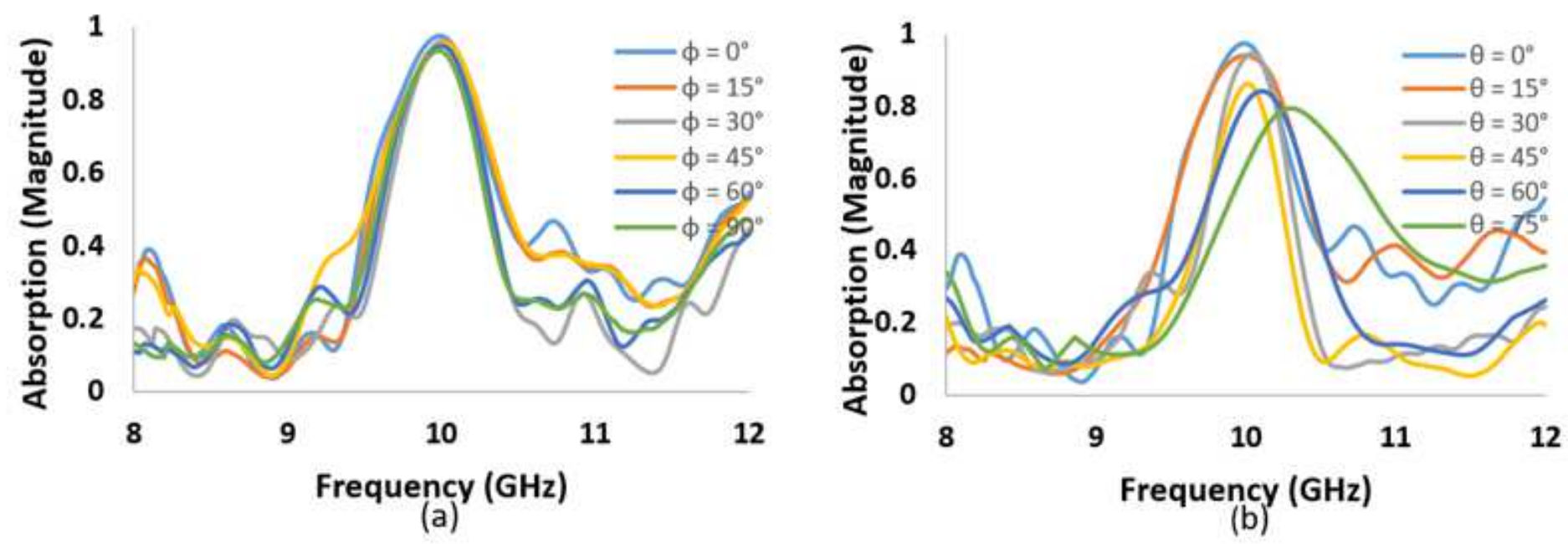

Figure 8

Simulated absorption (a) for different angle of polarization $\varphi$ and (b) for different incident angle $\theta$ 


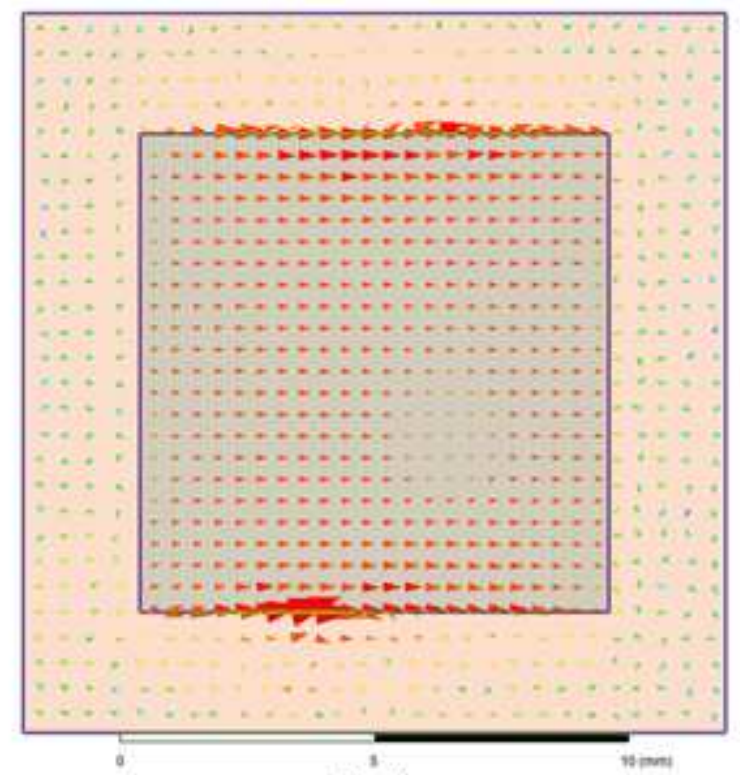

(a)

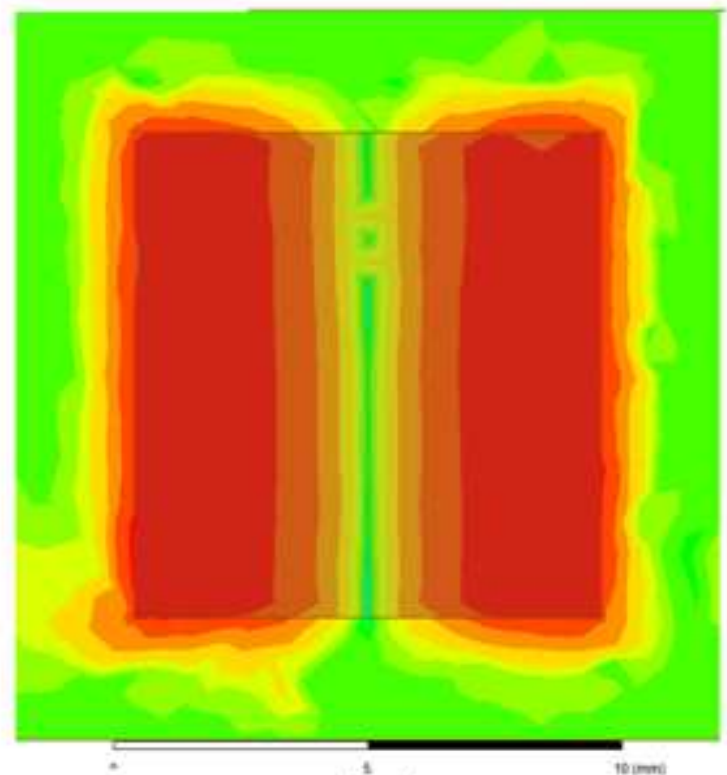

(b)

\section{Figure 9}

Simulated plot of (a) surface current distribution (b) electric field distribution of the proposed absorber at $10 \mathrm{GHz}$ 